\title{
The predictors of exercise capacity impairment in diabetic patients
}

\author{
Florina Frîngu ${ }^{12}$, Gabriel Guşetu ${ }^{12}$, Adriana Iosip ${ }^{12}$, Diana Gurzău ${ }^{12}$, Gabriela Dogaru1, Dumitru \\ Zdrenghea ${ }^{12}$, Dana Pop ${ }^{12}$ \\ 1. University of Medicine and Pharmacy "Iuliu Haţieganu” \\ 2. Department of Cardiology, Clinical Rehabilitation Hospital, Cluj-Napoca, Romania
}

\begin{abstract}
Background. The exercise capacity is a key issue in a diabetic patient's management, due to its well-known beneficial effects in terms of glycemic control, cardiovascular risk reduction and quality of life improvement. However the exercise capacity of diabetic patients is decreased many times and its determinants are sometimes less known. Our study aimed to assess the effort capacity in a cohort of diabetic patients and to find the main causative factors of its impairment.
\end{abstract}

Method: 61 patients with type-2 diabetes mellitus were enrolled and underwent and transthoracic echocardiography and a cycloergometer exercise testing. Exercise performance was calculated and the influence of clinical data and ultrasound parameters was assessed. Sedentary status of each patient was established from total time/week of at least moderate physical activity.

Results: the study group consisted of $48.4 \%$ women, mean age $61.4( \pm 8.4)$ years. Disease median duration was 5 years and $21.3 \%$ of the patients presented neuropathy, $4.5 \%$ retinopathy and $6.5 \%$ nephropathy. Exercise capacity was moderately and severe decreased ( $<5$ METs) in $37.7 \%$ of patients and in this subgroup the diastolic dysfunction, sedentary behavior and old age has a significantly higher prevalence. Interestingly, by multivariate regression, the sedentary lifestyle was the main determinant of decreased effort capacity (beta-coefficient 1.37, $\mathrm{p}<0.001$ ), suggesting the potential benefits of physical training in these patients.

Conclusion. Our study found a decreased effort capacity in at least one third of the patients and this is mainly due to sedentary lifestyle and deconditioning, the diastolic dysfunction also contributes to decreased effort capacity in diabetic patients.

Keywords: diabetes mellitus, exercise performance, diastolic dysfunction, exercise testing

\section{Introduction.}

Type 2 diabetes is a growing health issue in Western Countries and its and morbidity is mostly related to cardiovascular involvement. The disease is diagnosed in 366 billion people (56 million cases in Europe) and it is estimated to encounter about 522 billion in the year 2030 [1].

Regular physical play a key role not only in glycemic control [2], but also in improving lipidic profile [3], reducing visceral adipose tissue and insulin resistance and finally decreasing the cardiovascular risk (which is two times higher in the presence of diabetes) and mortality.

All the beneficial effects of exercise are independent of age and gender. They are reversible and reproducible [4].
Diabetes represents a unpleasant psychosocial situation that affects the quality of life due to insulin administration, dietary monitoring, blood sampling for glycemia measurement. The lower level of quality of life impaires the degree of hapiness, favors depression, decrease the labor participation rate and treatment compliance.

Accordingly the addition of a regular exercise regimen to drug treatment increases not only the life expectancy but also improves the quality of life $[5,6]$, which is of utmost importance in the global management of diabetes.

Nevertheless, the exercise level in diabetic persons is decreased and the causative factors identification play is a key point before the initiation of an educational 
program targeting the nonpharmacologic control of disease.

That's why the present study aims to assess through a standard exercise stress testing, the effort capacity in a cohort of diabetic patients without cardiovascular disease and to identify its main determinants.

\section{Method}

Patients. The study included 61 patients with type 2 diabetes mellitus (DM2) admitted in the Department of CardiologyRehabilitation Cluj-Napoca. All DM2 patients fulfilled the American Diabetes Association diagnosis criteria [7]. Patients with any signs, symptoms or ECG changes of a cardiovascular disease were excluded. We also ruled out the patients with neurological, respiratory, liver or kidney disease which could contraindicate or impair the exercise testing results. Patients with joint degenerative conditions limiting the proper cycling during testing were also excluded.

The study was conducted in accordance with the Declaration of Helsinki for human studies and was approved by the institutional ethical committee. All patients gave a signed informed consent to participate in the study.

Study design. Between Jan 2106 and December 2016 the patients were evaluated in terms of DM2 history, other cardiovascular risk factors (smoking, obesity, dyslipidemia, arterial hypertension), history for cardiovascular disease, a standard (12-lead) electrocardiography (ECG) and biochemistry data obtained by local laboratory. The sedentary status of each patient was established if total time of moderate physical activity was < $150 \mathrm{~min} /$ week . All patients were further evaluated by a standard Doppler echocardiography and an exercise stress testing on cicle-ergometer.

Echocardiography.

The

transthoracic echocardiography was performed on the left lateral decubitus using a $3.5 \mathrm{MHz}$ transducer on an Philips Affinity 70 echo machine. Long axis, short axis, two chamber and four chamber views were obtained in order to perform M-mode and B- mode (2D) measurements of chamber diameters and volumes and left ventricle ejection fraction (LVEF). LVEF was calculated using the modified Simpson's method from a four-chamber apical view.

Left ventricular diastolic performance was assessed by pulsed-wave Doppler measurement of the mitral E (early diastolic) and A (late diastolic) inflow velocities. The $\mathrm{E} / \mathrm{A}$ ratio and deceleration time (DT) of the early filling velocity were also recorded. Additional mitral annular septal early $\left(E_{s}^{\prime}\right)$ and late $\left(A_{s}^{\prime}\right)$ diastolic velocities were assessed by tissue Doppler imaging. The $\mathrm{E} / \mathrm{E}^{\prime}$ ratio was calculated in order to estimate the LV filling pressure and to distinguish a normal inflow pattern from pseudonormal LV filling (both with an E/A ratio $=0.8-1.5)$. $\mathrm{DD}$ was categorized in three stages [8]: normal (E/A ratio $=0.8-1.5$, $\left.\mathrm{DT}=160-200 \mathrm{~ms}, \mathrm{E}_{\mathrm{s}} \geq 8 \mathrm{~cm} / \mathrm{s}, \mathrm{E} / \mathrm{E}_{\mathrm{s}}{ }^{\prime}<8\right), 1^{\text {st }}$ degree (E/A ratio $<0.8$, DT $>200 \mathrm{~ms}, \mathrm{E}_{\mathrm{s}}<$ $\left.8 \mathrm{~cm} / \mathrm{s}, \mathrm{E} / \mathrm{E}_{\mathrm{s}}{ }^{\prime}<8\right), 2^{\text {nd }}$ degree $(\mathrm{E} / \mathrm{A}$ ratio $=$ $0.8-1.5, \mathrm{DT}=160-200 \mathrm{~ms}, \mathrm{E}_{\mathrm{s}}^{\prime}<8 \mathrm{~cm} / \mathrm{s}, \mathrm{E} / \mathrm{E}_{\mathrm{s}}{ }^{\prime}=$ 9-14), $3^{\text {rd }}$ degree (E/A ratio $>2$, DT $<160$ $\left.\mathrm{ms}, \mathrm{E}_{\mathrm{s}}^{\prime}<8 \mathrm{~cm} / \mathrm{s}, \mathrm{E} / \mathrm{E}_{\mathrm{s}}^{\prime} \geq 15\right)$. According to these parameters, the patients were assigned in one of the three DD stages.

Exercise stress testing. DM2 patients also performed a standard exercise stress testing on cycloergometer, using the 25W / 3 min step protocol. The maximal workload (W), blood pressure and heart rate, continuous 12-leads ECG monitoring were recorded and analyzed on a Cardiax 3.1 for Windows software. Maximum exercise capacity was calculated and expressed as METs.

Statistical analysis was carried out using the Microsoft Excel 2007 and SPSS for Windows ( $v$ 20.0, IBM Corporation, USA) software programs. The results were presented as numbers and percentages for qualitative variables and, for quantitative variables as mean \pm standard deviation; for nonparametric variables, median and range was used. In order to assess the normal (parametric) distribution of continuous numerical variables, the KolmogorovSmirnov test was used. To compare the 
qualitative variables (frequencies), the chisquared $\left(\chi^{2}\right)$ test was used. The analysis of quantitative variables with normal distribution was carried out using the Student t-test (t); for nonparametric variables the Mann-Whitney test was applied. A value of p $<0.05$ was considered statistically significant.

Results.

Demographic and personal history data are presented in table 1.

Table 1. The demographic data and the clinical characteristics of the patients IQR, interquartile range; $\mathrm{SD}$, standard deviation.

\begin{tabular}{cr}
\hline Treatment groups, n (\%) & $13(21.3)$ \\
\hline Diet only & $39(63.4)$ \\
Oral medication & $11(18)$ \\
Insulin & $3(4.5)$ \\
\hline Disease complications, n (\%) & $4(6.5)$ \\
Retinopathy & $13(21.3)$ \\
Nephropathy & $3(4.5)$ \\
Neuropathy & \\
Multiple & $60(98.3)$ \\
\hline Associated CVRF, n (\%) & $13(21.3)$ \\
\hline Hypertension & $10(16.4)$ \\
Dyslipidemia & $40(65.5)$ \\
Smoking & $24(39.3)$ \\
Obesity & $13(21.3)$ \\
Class I & $3(5)$ \\
Class II & \\
Class III & $26(42.6)$ \\
\hline Sedentary status, n (\%) & $35(57.4)$ \\
\hline Active &
\end{tabular}

The complications were recorded in 4.5\% (retinopathy), 6.5\% (nephropathy) and $21.3 \%$ (neuropathy).

Among cardiovascular risk factors hyper-tension encountered a 98.3\% and obesity $65.5 \%$ (table 1 ), both of them recognized to impair exercise performance. patients, $18 \%$ on insulinotherapy (alone or combination) and $21.3 \%$ of them followed diet treatment only.

Table 2. The main parameters of cycloergometer exercise testing

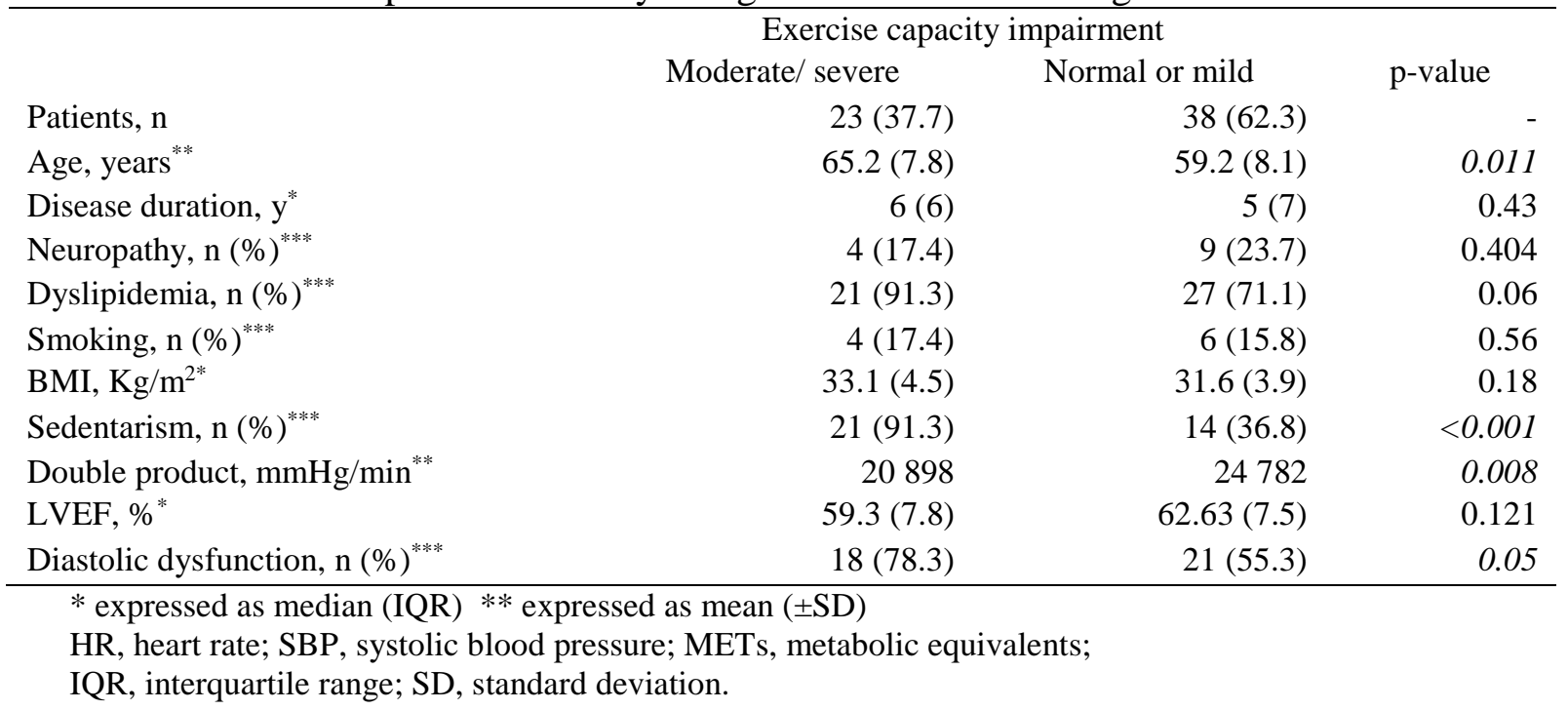


The exercise testing parameters are presented in table 2 and generally reflect a slight decrease of effort performance (mean value 5.3 METs). Based on calculated effort capacity (METs) we divided the patients into two subgroups, with moderate-to-severe impaired exercise capacity ( $<5$ METs) and with normal or mild decreased effort capacity ( $\geq 5 \mathrm{METs}$ ) (figure 1 ).

The mean age, diastolic dysfunction prevalence and sedentary status were significantly different in the two subgroups (table 3). Conversely, the diabetes duration, and other cardiovascular risk factors did not differ among these two subgroups. De double product (max blood pressure multiplied by max heart rate) was significantly decreased in the first subgroup $(p=0.008)$, even if the mean value was above 22000 in the entire studied group.

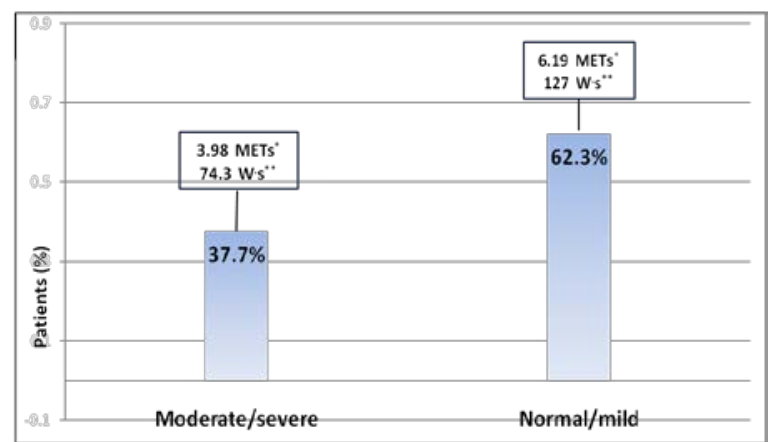

Figure 1. The distribution of the patients in the two subgroups ("moderately to severe impaired" vs "preserved" effort capacity) and main exercise test parameters

Table 3. The correlation between exercise capacity, disease characteristics and cardiac ultrasound parameters

\begin{tabular}{|c|c|c|c|}
\hline & \multicolumn{2}{|c|}{ Exercise capacity impairment } & \multirow[b]{2}{*}{ p-value } \\
\hline & Moderate/ severe & Normal or mild & \\
\hline Patients, $\mathrm{n}$ & $23(37.7)$ & $38(62.3)$ & 1 \\
\hline Age, years ${ }^{* *}$ & $65.2(7.8)$ & $59.2(8.1)$ & 0.011 \\
\hline Disease duration, $\mathrm{y}^{*}$ & $6(6)$ & $5(7)$ & 0.43 \\
\hline Neuropathy, n (\%) & $4(17.4)$ & $9(23.7)$ & 0.404 \\
\hline Dyslipidemia, n (\%) ${ }^{* * *}$ & $21(91.3)$ & $27(71.1)$ & 0.06 \\
\hline Smoking, $\mathrm{n}(\%)^{* * *}$ & $4(17.4)$ & $6(15.8)$ & 0.56 \\
\hline $\mathrm{BMI}, \mathrm{Kg} / \mathrm{m}^{2 *}$ & $33.1(4.5)$ & $31.6(3.9)$ & 0.18 \\
\hline Sedentarism, $\mathrm{n}(\%)^{* * *}$ & $21(91.3)$ & $14(36.8)$ & $<0.001$ \\
\hline Double product, $\mathrm{mmHg} / \mathrm{min}^{* *}$ & 20898 & 24782 & 0.008 \\
\hline LVEF, \%* & $59.3(7.8)$ & $62.63(7.5)$ & 0.121 \\
\hline Diastolic dysfunction, $\mathrm{n}(\%)^{* * *}$ & $18(78.3)$ & $21(55.3)$ & 0.05 \\
\hline
\end{tabular}

In order to assess the strength of each of the three determinants (age, diastolic dysfunction and sedentary status) we performed a multivariate linear regression analysis and the sedentary status seems to be the main factor related with impaired exercise performance (betacoefficient=1.3- table 4). Moreover, the diastolic dysfunction has similar prevalence in the subgroup of sedentary and active patients (figure 2).

Table 4. The multivariate linear regression analysis for exercise capacity determinants.

\begin{tabular}{lccc}
\hline Variable & B-coefficient & Std. Error for B & Significance \\
(Constant) & 7.827 & 1.046 & 0.000 \\
Sedentarism & 1.376 & .267 & 0.000 \\
Diastolic dysfunction & -.715 & .217 & 0.002 \\
Age & -.041 & .016 & 0.012 \\
\hline
\end{tabular}

Dependent Variable: METs 
Figure 2. The diastolic dysfunction prevalance is similar among sedentary and active individuals. Active individuals have a better execise capacity

\section{Discussion.}

Our study has shown the effort capacity is significantly decreased in more than one third of diabetic patients without clinical signs and ECG evidences of cardiac disease. Ivanova et al also reports a decreasd exercise participation among diabetics as a consequence of anxiety or depression, common among these patients. Further, less exercise predicts worsening of symptomatology of depression [9].

Increased age is one of the factors significantly different in the subgroup with impaired exercise capacity and this is recognized as a limiting factor in general population. Central mechanisms involving cardiovascular (arterial stiffening, increased pulse pressure, decreased endothelium-mediated vasodilatation, increased left ventricular wall rigidity, prolonged myocardial contraction, prolonged early diastolic filling rate, decreased conduction times, sclerosis and calcification of the valves, autonomic nervous system changes) [10] and respiratory system [11] along with peripheral changes (sarcopenia) [12] represent a part of the background mechanisms decreasing effort capacity in elderly.

Surprisingly, the smoker status also did not correlate with decreasing of effort capacity probably due to small number of smoker (10 patients). In one study designed on 42 smokers and 50 nonsmokers, the autors have shown a greater rate-pressure product during exercise in smokers (due to significantly higher systolic blood pressure), a higher submaximal heart rate and finally, an impaired exercise tolerance and significantly

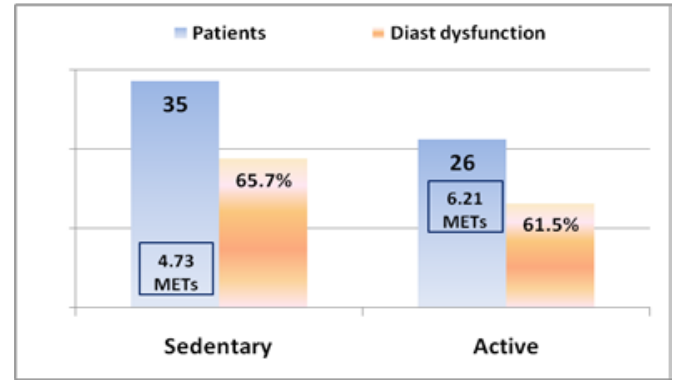

shorter maximal exercise test duration [13].

The exercise performance did not correlated with other cardiovascular risk factors, even if for dyslipidemia the statistical significance was at the border $(p=0.06)$. The number of the patients could explain again the result. Hypertension, a well-known contributing factor to left ventricular hypertrophy, increased arterial rigidity and through these, to decreasing of physical performance, was not taken into aaccount, because it was prezent in all patients except one [14].

Another unexpected result was the lack of correlation between decreased effort tolerance and the disease duration. The history of diabetes is well-known to correlate with decreasing quality o life through the increasing risk of diabetes complications. All of these complications correlate also with exercise capabilities. Janevic et al have shown that diabetes patients with complications (microvascular or cardiovascular) are less likely to reach the recommended physical activity levels compared to those without complications [15]. These patients require additional support to perform the recommended levels of physical activity. In our study the eterogenity of the group in terms of diabetes treatment and the reduced prevalance of complication may influence this contradictory finding. The hypothesis is supported by the absence of correlation between diabetic neuropathy and poor effort tolerance among our group.

Left ventricle ejection fraction was also normal in all subjects and did not influence their effort capacity. The double product (systolic blood pressure multiplied 
by heart rate) was above $22000 \mathrm{~W} \cdot \mathrm{s} / \mathrm{min}$ and could suggest a good mechanical work of the heart. The double product was however decreased among patients with decreased effort capacity and this supports the involvement of cardiovascular system at least in part to the decreasing of exercise capacity. Diastolic dysfunction was registered in $63.3 \%$ of patients and represented an important determinant of limiting exercise performance. The observation converge with the results of Bajraktari et al in a study conducted in 200 patients where diastolic dysfunction was found in $68 \%$ of the diabetic patients [16]. The mechanism of diastolic dysfunction among diabetes, even if extensively studied, is not yet completely known; Early or accelerated changes into extracellular matrix, interstitial fibrosis or autonomic system involvement could result in the onset of diastolic dysfunction. As a result, asymptomatic patients at rest may be symptomatic with exercise and this further may decrease the duration of "free of symtoms" exercise which limit the patient's daily activities and his quality of life

The multivariate regression analysis for effort capacity determinants has however shown the sedentary status overweighs the diastolic dysfunction in determination the effort intolerance. Moreover, the diastolic dysfunction was similar among sedentary and active patients even if sedentary patients have a significantly lower exercise performance.

The sedentary behavior in diabetes is a common issue [17] and factors contributing to sedentary lifestyle are many [18]; their control was demonstrated to improve the quality of live and the outcome of these patients [19]. Furthermore, these benefits could be enhanced y physical training. According to current literature, endurance training consisting of moderate - to vigorous intensity aerobic exercise (e.g., walking, cycling, etc.), 30-60 min duration, 5 days/ week [20] or $150 \mathrm{~min} /$ week, be performed with $50-70 \%$ of maximum heart rate [21], is expected to improve glycemic control [22], not only mediated by weight loss but also by increase in muscle sensitivity; physical training also demonstrated a better control of lipidic profile, hemodinamic parameters - diastolic performance, stroke volume, exercise blood pressure by improvement of endothelium mediated vasodilatation - and beneficial effect on cognition and on depression symptoms.

Study limitations. Given the retrospective character of our research, it is possible that some information to miss despite efforts to record all useful information. The limited dimensions of study group could lead for some of the results not to reach the statistical significance. The using of cycle-ergometer to perform exercise testing is less accurate than treadmill in terms of assessing the effort capacity neglecting the weight of the subject; this was however taken into account by testing software when calculated the effort capacity. Finally, the sedentary status of the patient was established by a self -assessment and this may have introduced bias. However the exact mentioning of time spent for certain physical activities warranted us to collect as homogenous data as possible.

In conclusion, the exercise capacity is moderate to severe decreased in at least one third of diabetic patients without overt cardiac disease and this is mainly due to their sedentary status and to diastolic dysfunction of the left ventricle. The data supports the need for educational and training programs in this category, in order to improve their quality of life and prognosis.

\section{REFERENCES}

1. Zimmet PZ, McCarty DJ, de Courten MP. The global epidemiology of non-insulin-dependent diabetes mellitus and the metabolic syndrome. J Diabetes Complications 1997;11:60-8.

2. Sigal RJ, Kenny GP, Wasserman DH, Castaneda-Sceppa C. Physical activity/exercise and type 2 diabetes. Diabetes Care 2004;27:2518-39.

3. Thomas DE, Elliott EJ, Naughton GA. Exercise for type 2 diabetes mellitus. Cochrane Database Syst Rev 2006;19. 
4. Francesconi C, Lackinger C, Weitgasser R, Haber P, Niebauer J. [Physical activity and exercise training in the prevention and therapy of type 2 diabetes mellitus]. Wien Klin Wochenschr 2016;128:015-0923.

5. Snel M, Sleddering MA, Vd Peijl ID et al. Quality of life in type 2 diabetes mellitus after a very low calorie diet and exercise. Eur J Intern Med 2012;23:143-9.

6. Çolak TK, Acar G, Dereli EE et al. Association between the physical activity level and the quality of life of: J Phys Ther Sci. 2016 Jan;28(1):142-7. Epub 2016 Jan 30 doi:10.1589/jpts.28.142.

7. Standards of Medical Care in Diabetes-2016: Summary of Revisions. Diabetes Care 2016;39:dc16-S003.

8. Nagueh SF, Appleton CP, Gillebert TC et al. Recommendations for the evaluation of left ventricular diastolic function by echocardiography. Eur J Echocardiogr 2009;10:165-93.

9. Ivanova E, Burns RJ, Deschenes SS, Knauper B, Schmitz N. A Longitudinal Investigation of Anxiety and Depressive Symptomatology and Exercise Behaviour Among Adults With Type 2 Diabetes Mellitus. Can J Diabetes 2017;41:73-81.

10. Lakatta EG, Wang M, Najjar SS. Arterial aging and subclinical arterial disease are fundamentally intertwined at macroscopic and molecular levels. Med Clin North Am 2009;93:583-604.

11. Dubois P, Lulling J, Delwiche JP, Bouckaert A, Prignot J. [Respiratory factors limiting exercise capacity in elderly patients (author's transl)]. Bull Eur Physiopathol Respir 1979;15:385-94.

12. Are deferrable the mobility impairments in older aging?: Eur J Transl Myol. 2016 Feb 23;26(1):5831. doi:10.4081/ejtm.2016.5831.

13. Papathanasiou G, Georgakopoulos D, Georgoudis G, Spyropoulos P, Perrea D, Evangelou A. Effects of chronic smoking on exercise tolerance and on heart rate-systolic blood pressure product in young healthy adults. Eur J Cardiovasc Prev Rehabil 2007;14:646-52.

14. Guirado GN, Damatto RL, Matsubara BB et al. Combined exercise training in asymptomatic elderly with controlled hypertension: effects on functional capacity and cardiac diastolic function. Med Sci Monit 2012;18:CR461-5.

15. Janevic MR, McLaughlin SJ, Connell CM. The association of diabetes complications with physical activity in a representative sample of older adults in the United States. Chronic Illn 2013;9:251-7.

16. Bajraktari G, Qirko S, Bakalli A, Rexhepaj N, Elizi S. Reduced left ventricular diastolic function in asymptomatic patients with non-insulindependent diabetes mellitus. Med Arh 2004;58:339-41.

17. Cichosz SL, Fleischer J, Hoeyem $\mathrm{P}$ et al. Objective measurements of activity patterns in people with newly diagnosed Type 2 diabetes demonstrate a sedentary lifestyle. Diabet Med 2013;30:1063-6.

18. Rockette-Wagner B, Edelstein S, Venditti EM et al. The impact of lifestyle intervention on sedentary time in individuals at high risk of diabetes. Diabetologia 2015;58:1198-202.

19. Long-term effects of lifestyle intervention or metformin on diabetes development and microvascular complications over 15-year followup: the Diabetes Prevention Program Outcomes Study. Lancet Diabetes Endocrinol 2015;3:866-75. 20. Zdrenghea D, Pop D, Penciu O, Zdrenghea V, Zdrenghea M. Rehabilitation in diabetic patients. Rom J Intern Med 2009;47:309-17.

21. Piepoli MF, Hoes AW, Agewall S et al. 2016 European Guidelines on cardiovascular disease prevention in clinical practice: The Sixth Joint Task Force of the European Society of Cardiology and Other Societies on Cardiovascular Disease Prevention in Clinical Practice (constituted by representatives of 10 societies and by invited experts)Developed with the special contribution of the European Association for Cardiovascular Prevention \& Rehabilitation (EACPR). Eur Heart J 2016;37:2315-81.

22. Ruffino JS, Songsorn $\mathrm{P}$, Haggett $\mathrm{M}$ et al. A comparison of the health benefits of reduced-exertion high-intensity interval training (REHIT) and moderate-intensity walking in type 2 diabetes patients. Appl Physiol Nutr Metab 2017;42:202-208 